\title{
Validación de la versión española de la Gender Role Conflict Scale for Adolescents (GRCS-A)
}

\author{
Elisa Larrañaga ${ }^{1 *}$, Santiago Yubero $^{1}$, Anastasio Ovejero ${ }^{2}$ y Raúl Navarro ${ }^{1}$
}

${ }^{2}$ Universidad de Valladolid

${ }^{1}$ Universidad de Castilla-La Mancha

\begin{abstract}
Resumen: La Gender Role Conflict Scale for Adolescents (GRCS-A) mide el conflicto de rol de género de los adolescentes. La GRCS-A se compone de cuatro factores referidos a distintos patrones de conflicto (afecto limitado entre hombres, emotividad restringida, conflicto entre trabajo, escuela y familia, y necesidad de éxito y logro). El objetivo de este estudio es analizar la estructura factorial de la versión española de la GRCS-A y la congruencia factorial con los datos de la versión inglesa. Nuestra muestra está compuesta por 1595 estudiantes varones de Enseñanza Secundaria y Bachillerato de diferentes centros educativos de Castilla-La Mancha y Castilla y León (España). Se examinó la correlación de la escala con el sexismo y el acoso escolar, y se analizaron las diferencias de acuerdo a la edad en la experimentación de los distintos patrones de conflicto. Los análisis factoriales, exploratorio y confirmatorio, replicaron la estructura factorial de la versión original y las correlaciones mostraron que el conflicto de rol de género está relacionado con el sexismo y el acoso escolar. El análisis de regresión mostró la influencia de la edad en el conflicto de rol de género.

Palabras clave: Conflicto de rol de género; estructura factorial; adolescentes; sexismo; acoso escolar; validación.
\end{abstract}

Title: Validation of the Spanish version Gender Role Conflict Scale for Adolescents (GRCS-A).

Abstract: Gender role conflict scale for adolescents (GRCS-A) measures the gender role conflict experienced by adolescents. GRCS-A consists of four patterns, namely Restricted affection between men, Restricted emotionality, Conflict between work, school, and family, and Need for success and achievement. The objective of this study was to analyze the factorial structure of the Spanish version of GRCS-A and the factorial congruence with the original version using a sample of 1595 male adolescents from Castilla-La Mancha and Castilla y León (Spain). Correlations between GRCS-A and sexism and bullying were examined. Age differences in the experimentation of gender role conflict patterns were also analyzed. Exploratory and confirmatory factorial analysis replicated the original factorial structure and correlation analysis showed that Gender role conflict is related to sexism and bullying. Regression analysis showed a significant effect of age over the gender role conflict.

Keywords: Gender role conflict; factorial structure; adolescents; sexism: bullying; validation.

\section{Introducción}

Desde hace años se está produciendo una creciente discusión en torno a la naturaleza restrictiva de la socialización de género y su influencia sobre la salud y el bienestar psicológico de los individuos (Blazina, Pisseco y O’Neil, 2005) entendiendo que algunos de los valores de género tradicionales son disfuncionales y contradictorios. Concretamente, la adhesión rígida a los valores y estándares de género tradicionales puede llegar a provocar un conflicto de naturaleza tanto interna como interpersonal en aquellos varones que, habiendo interiorizado rasgos y roles estereotipados, sienten que sus conductas, actitudes e intereses no se adecúan a sus propias creencias (O’Neil, 1990), lo que se conoce como Conflicto de Rol de Género (GRC, Gender Role Conflict). Aparece cuando los roles de género rígidos, sexistas o restrictivos provocan una limitación personal y una desvalorización de uno mismo o de otras personas (O’Neil, Helms, Gable, David y Wrightsman, 1986).

La investigación en torno al GRC ha cobrado especial importancia en los estudios de género, debido a las repercusiones de este conflicto en la salud mental de los hombres (ver O’Neil, 2008 para una revisión). A nivel interpersonal, el GRC se ha vinculado con las actitudes hostiles y estereotipadas hacia mujeres (Robinson y Schwartz, 2004), machismo (Liang, Salcedo y Miller, 2011), problemas en las relaciones entre iguales (Groeschel, Wester y Sedivy, 2010) y con conductas agresivas (Cohn, Jakupcak, Seibert, Hildebrandt y Zeichner, 2010).

\section{* Dirección para correspondencia [Correspondence address]:}

Elisa Larrañaga. Departamento de Psicología. Edificio Melchor Cano. Campus Universitario. 16071 Cuenca (España).

E-mail: Elisa.Larranaga@uclm.es
Para investigar este constructo, O’Neil et al (1986) desarrollaron la Escala de Conflicto de Rol de Género Masculino (Gender Role Conflict Scale - GRCS). La GRCS ha sido traducida a más de 10 idiomas y ha sido utilizada para medir el Conflicto de Rol de Género en distintos países (Australia, Portugal, Corea, Japón, Suecia, Alemania, Canadá o Francia). El GRC puede comenzar en la adolescencia y experimenta cambios a lo largo del ciclo vital (Watts y Borders, 2005). Sin embargo, los estudios sobre el GRC en diferentes grupos de edad son incipientes. Para estudiar los patrones de conflicto durante la adolescencia, Blazina et al. (2005) realizaron la adaptación de la GRCS a esta etapa del desarrollo, creando la Gender Role Conflict Scale - Adolescent Version (GRCS-A). Para ello, modificaron los ítems de la escala original para hacerlos más apropiados al desarrollo evolutivo de la etapa adolescente manteniendo la consistencia teórica con el constructo original. Concretamente, reescribieron los ítems para ajustarse a las relaciones personales ("Cuando me involucro personalmente con otros, no expreso mis sentimientos más fuertes"). Incluyeron, además, actividades escolares considerándolas como una fuente potencial de conflicto y de competición entre los adolescentes ("Para mí es importante conseguir el primer puesto de mi clase") y adecuaron la presentación lingüística de los ítems al nivel de competencia lectora de la etapa adolescente. En su estudio, Blazina et al. (2005) encontraron una estructura factorial similar a la obtenida con las muestras de adultos, identificando cuatro patroHombres (Restricted Affection Between Men, RABM); Emotividad Restringida (Restricted Emotionality, RE), Conflicto entre Trabajo, Escuela y Familia (Conflict Between Work, School, and Family, CWSF); y Necesidad de Éxito y Logro (Need for Success and Achievement, NSA). El primer patrón de conflicto nes de conflicto que denominaron: Afecto Limitado entre 
(RABBM) implica restricciones para expresar las emociones e ideas personales a personas del mismo sexo e incluso la dificultad para tocar a otros hombres. Este patrón de conflicto está apoyado en el mantenimiento de mitos y estereotipos que dificultan el apoyo entre los hombres. El segundo patrón (RE) es una limitación a la hora de compartir los estados emocionales propios. Enfatiza el miedo a expresar los propios sentimientos, así como la dificultad para encontrar palabras que puedan expresar las emociones básicas. Se trata de un patrón de conflicto que supone una limitación en la expresión conductual y verbal hacia los otros. El tercer patrón (CBWFR) refleja las dificultades para equilibrar las responsabilidades entre los distintos dominios de la vida, centrados en el trabajo y los requerimientos de la escuela respecto a las relaciones familiares. El último patrón de conflicto (NSA) involucra un temor persistente sobre el propio éxito, la obtención de poder y el estatus social en comparación con otros. Este patrón describe el grado en que los chicos han sido socializados en la obtención del éxito a través de la competencia. Blazina et al. (2005) sugieren que esta subescala es un reflejo de los aspectos positivos de la ideología de género. Los cuatro factores explicaban el $40.9 \%$ de la varianza total y tenían coeficientes de consistencia interna que oscilaban entre 0.72 y el 0.82 .

$\mathrm{Al}$ igual que en los adultos, el GRC se asocia con el malestar psicológico en los adolescentes (Fagot, Rodgers y Leinbach, 2000; Kiselica, 2011; O’Neil, Good y Holmes, 1995). La GRCS-A correlaciona significativamente con la depresión (Choi, Kim, Hwang y Heppner, 2010), la ansiedad (Kim, Choi, Kim y Park, 2009) y la resiliencia (BatesGalligan, Barnett, Brennan y Israel, 2010). A nivel interpersonal el CRG en adolescentes se ha vinculado con actitudes tradicionales hacia la mujer (Addelston, 1995) y con el incremento de conflictos en la escuela y en la familia (Watts y Borders, 2005).

Esta escala a pesar de ser un instrumento válido para medir la experimentación del conflicto de rol de género entre adolescentes no ha recibido la misma atención por parte de la comunidad internacional que la versión para adultos. Recientemente se ha realizado su adaptación al coreano (Kim, Choi, Kim y Park, 2009) pero hasta hoy no había sido validada en el contexto español. La finalidad de este estudio instrumental (Carretero-Dios y Pérez, 2007; Montero y León, 2007) ha sido la de analizar las propiedades psicométricas de la GRCS-A (Blazina et al., 2005) en una muestra española. Para ello se analizaron las propiedades métricas de los ítems que componen la escala, sometiendo a examen su estructura factorial mediante análisis factorial exploratorio (AFE), que fue contrastada mediante un análisis factorial confirmatorio (AFC). Una vez confirmada la estructura, se analizó la fiabilidad de consistencia interna, así como diversos indicadores de validez de sus medidas. Para proporcionar evidencias de la validez concurrente de la adaptación española, se optó por seleccionar variables de relaciones interpersonales. Concretamente se examinó la correlación de la GRCS-A con el sexismo ambivalente y el acoso escolar es- perando encontrar una relación positiva del GRC con ambas variables. El sexismo y el acoso escolar fueron seleccionados considerando la relación encontrada por la investigación previa entre el GRC y el sexismo (Covell, 1988; Schwartz et al., 2005) y también con la conducta agresiva (Cohn, Zeichner and Seibert, 2008). Entre las conductas agresivas y violentas se analizó la asociación entre el GRC y el acoso escolar atendiendo al llamamiento realizado por O’Neil (2008) sobre la necesidad de examinar la influencia del GRC sobre esta forma de agresión más habitual durante la adolescencia.

Blazina $(1997,2001)$ y O’Neil et al (1995) teorizaron que el conflicto de rol de género evoluciona con la edad. Sin embargo, son pocos los estudios que analizan su influencia (O’Neil, 2008). Un objetivo adicional de este estudio consistió en analizar la influencia de la edad sobre las dimensiones del GRC en la adolescencia.

En síntesis, el primer objetivo de este estudio fue confirmar la estructura factorial del GRCS-A en adolescentes españoles y evaluar la consistencia de sus escalas. En segundo lugar, analizar su vinculación con el sexismo y con la agresión entre iguales en el contexto escolar. En tercer lugar, se realizó un análisis de regresión tomando como variable predictora la edad.

\section{Método}

\section{Participantes}

La muestra constó de 1595 varones que completaron todos los ítems de los cuestionarios. Los participantes eran estudiantes de Educación Secundaria Obligatoria (ESO) y Bachillerato, procedentes de 27 institutos públicos. El 87.6\% es de origen español. La edad media de los participantes es de 14.49 años $(D T=1.46)$. La distribución por curso fue de $15.1 \%, 24.6 \%, 24.1 \%$ y $16.4 \%$ para primero, segundo, tercero y cuarto de ESO respectivamente, $19.7 \%$ estudiantes de Bachillerato.

\section{Instrumentos}

Se utilizaron principalmente tres instrumentos. En primer lugar, la Escala de Conflicto de Rol de Género para Adolescentes (GRCS-A, Blazina et al., 2005) (la exploración de las consecuencias negativas de la adhesión a los roles de género tradicionales se ha realizado mediante la adaptación para adolescentes de la Gender Role Conflict Scale).

Esta escala está constituida por 29 enunciados en los que los adolescentes informan de su acuerdo o desacuerdo con cada enunciado en una escala tipo Likert con un rango de 1 (muy en desacuerdo) a 6 (muy de acuerdo). La estructura factorial reproduce tres de los factores del modelo adulto: Emotividad Restringida (RE, Restricted Emotionality) con 9 ítems, por ejemplo: "No me gusta mostrar mis emociones a otras personas"); Afecto Limitado entre Hombres (RABM, Restricted Affection Between Men) con 7 ítems, por ejemplo "Me resulta difícil abrazar a otros hombres"); Conflicto entre 
Trabajo, Escuela y Familia (CWSF, Conflict Between Work, School, and Family) con 7 ítems, por ejemplo "Me resulta difícil encontrar tiempo para descansar". Sin embargo, en la escala para adolescentes emerge un nuevo factor, Necesidad de Éxito y Logro (NSA, Need for Success and Acbievement) con 6 ítems, por ejemplo "Ganar dinero es parte de mi idea de lo que es ser un hombre de éxito") que es reflejo de aspectos positivos de la ideología masculina más que del conflicto de rol de género. Cuanto mayor es la puntuación en cada una de las subescalas, mayor es el conflicto de rol de género.

Los índices de fiabilidad informados por Blazina et al. (2005) para cada una de las subescalas son: Necesidad de Éxito y Logro, $\alpha=.95$; Emotividad Restringida, $\alpha=.87$; Afecto Limitado entre Hombres, $\alpha=.83$; y Conflicto entre Trabajo, Escuela y Familia, $\alpha=.60$.

También utilizamos la Escala de Sexismo Ambivalente (Glick y Fiske, 1996; adaptación española de Expósito, Moya y Glick, 1998). Esta escala está compuesta por 22 ítems organizados en dos subescalas. La Escala de Sexismo Hostil está constituida por 11 ítems referidos a actitudes tradicionales y prejuiciosas hacia las mujeres, basadas en la supuesta inferioridad de las mismas (por ejemplo: "La mayoría de las mujeres no aprecia completamente todo lo que los hombres hacen por ellas"). La Escala de Sexismo Benévolo también consta de 11 ítems que expresan actitudes tradicionales hacia los roles de género, enfatizan la debilidad y necesidad de protección de las mujeres, aunque desde un tono afectivo positivo (por ejemplo: "Aun cuando un hombre logre muchas cosas en su vida, nunca podrá sentirse verdaderamente completo a menos que tenga el amor de una mujer"). Cada uno de los ítems posee un rango de respuesta tipo Likert que va de 0 (totalmente en desacuerdo) a 5 (totalmente de acuerdo). Cuanto mayor es la puntuación, mayor es el nivel de sexismo que caracteriza a los participantes.

El coeficiente de fiabilidad de la escala total para este estudio fue de .84 , para la escala de sexismo hostil fue de .79 , y en la escala de sexismo benévolo fue de .78 .

En tercer lugar, del Cuestionario de Acoso Escolar (CAME), se emplearon los ítems que miden el acoso escolar entre iguales desde el puto de vista del agresor del "Instrument to assess the incidence of involvement in bully/victim interaction at school" (Rigby y Bagshaw, 2003). Los participantes debían responder si durante el pasado año habían participado de la agresión repetida a otras personas. La escala recoge información sobre distintas formas de acoso en contextos educativos, concretamente, agresión física ("He pegado o empujado a alguien"), agresión verbal ("He hablado de alguien a sus espaldas o he dicho cosas falsas sobre ellos") y exclusión social del grupo de iguales ("He ignorado o no he dejado participar a alguien de algunas actividades o juegos"). El cuestionario está compuesto por 5 ítems con formato de respuesta tipo Likert de 4 puntos ( $0=$ Nunca y $3=$ Diariamente). La consistencia interna para la escala sobre el rol de agresor fue de .79, para este estudio.

\section{Procedimiento}

En primer lugar, se procedió a la adaptación lingüística de la GRCS-A empleándose para ello la traducción hacia delante (Hambleton, 1996). Primero se tradujeron los ítems al castellano, dicha traducción se entregó, junto al original en inglés, a un experto bilingüe que recomendó una serie de cambios. Se modificó el original con los cambios sugeridos. A continuación se envío el cuestionario a cuatro jueces expertos en género, quienes juzgaron la adecuación y comprensión de los ítems, proponiendo una redacción alternativa en algún ítem que fue tomada en cuenta. El cuestionario final se pasó en un aula de ESO para que juzgaran la comprensión de los ítems. Se revisaron los ítems que contaban con un consenso de los alumnos inferior al $85 \%$, obteniéndose de esta forma la versión final del instrumento.

Una vez conseguido el consentimiento por parte de los centros escolares, se obtuvo el consentimiento pasivo de los padres y/o madres de los menores para que participaran en el estudio. Los padres y madres recibieron una carta en la que se les explicaba la naturaleza del estudio y se les informaba de su derecho a no autorizar la participación de su hijo. Ningún padre se negó a consentir la participación de su hijo. La batería de instrumentos se aplicó al principio de curso en cada uno de los grupos dentro del aula durante la hora de tutorías, sin que el profesor estuviera presente. El proceso fue supervisado por los investigadores. Se entregó un cuadernillo con los cuestionarios a cada alumno. A todos se les proporcionó las mismas instrucciones, incidiendo en el carácter voluntario, anónimo y confidencial de la investigación.

\section{Análisis estadísticos}

En primer lugar, para comprobar si se confirma el modelo multidimensional en los datos españoles, se realizó un análisis factorial usando rotación oblicua, especificando el número de factores en 4 , siguiendo el mismo modelo propuesto por los autores en la población adolescente estadounidense (Blazina et al., 2005).

Optamos por emplear también técnicas estructurales para llevar a cabo una aproximación más potente de la validación de la escala ya que esta técnica se ajusta mejor a los criterios señalados por Messick (1995) respecto a la validez de constructo. Se empleó el programa LISREL8.54 (Jöreskog y Sörbom, 1993), en el 50\% de la muestra, para confirmar el análisis factorial (AFC). Se ha empleado el procedimiento de Máxima Verosimilitud para la estimación, siguiendo el mismo modelo que en la adaptación al coreano de este cuestionario (Kim et al., 2009). Los índices considerados para evaluar el ajuste del modelo han sido el Goodness of Fit Index (GFI; Tanaka y Huba, 1985), el Adjusted Goodness of Fit Index (AGFI), el Comparative Fit Index (CIF) y el Root Mean Square Error Aproximation (RMSEA; Browne y Cudeck, 1989; Steiger, 1990). Para el GFI, el AGFI y el CIF se consideran valores por encima de .90 como adecuados. El RMSEA es 
considerado mejor indicador de ajuste global (Marsh, Balla y Hau, 1996). Para este indicador se considera que valores inferiores a .05 son óptimos; si bien, Browne y Cudeck (1993) consideran que valores entre .05 y .08 indicarían un ajuste aceptable, mientras que valores por encima de .10 indicarían un ajuste deficiente.

En segundo lugar, se calculó la consistencia interna de cada uno de los factores contenidos en la GRCS-A, mediante el estadístico alpha de Cronbach. En tercer lugar, se obtuvieron evidencias externas de la validez de la escala mediante correlaciones con las pruebas de sexismo ambivalente y la medida de acoso escolar. Por último, se realizó un análisis de regresión simple tomando como variable predictora la edad, empleando el método paso a paso.

\section{Resultados}

\section{Análisis de ítems}

El objetivo principal de esta fase fue detectar ítems con dificultades de comprensión. La mayor discusión se produjo en la traducción de determinados términos que no tienen un mismo significado en español. Por ejemplo, en el ítem 3 'ove' fue sustituido por 'cariño' ya que los adolescentes entendían por 'amor' exclusivamente una relación de pareja. En otros términos se empleó un sinónimo ya que los jueces los consideraron más apropiados para los adolescentes. Se mantuvieron las opciones que alcanzaron un elevado consenso entre los adolescentes, priorizando la adaptación sobre la literalidad del término (por ejemplo, 'time to relax': tiempo para descansar; 'hectic work': ajetreado trabajo, 'makes me feel anxious': me hace sentir incómodo).

Los resultados cuantitativos muestran que todas las opciones de respuesta son elegidas en todos los ítems. La media de respuesta de los ítems se sitúa entre 2.51 y 4.00 y las desviaciones típicas oscilan entre 1.54 y 1.91 , por lo que podemos suponer una adecuada variabilidad de las puntuaciones. Excepto los ítems 12 y 16 todas las correlaciones ítemtotal corregidas superan el valor de .30 (Nunnally y Bernstein, 1995). Pese a que se observa que la eliminación de estos ítems no mejora el alfa global de la escala (.87), se estudió la conveniencia de eliminar estos ítems en función de los otros resultados de la escala. Se presenta también la puntuación total de la escala como sumatorio, siguiendo a los autores originales de la misma (véase Tabla 1).

\section{Análisis factorial exploratorio (AFE)}

Para analizar la estructura factorial se realizó un análisis factorial de componentes principales con rotación oblicua Promax, como en la adaptación original para adolescentes. Como criterio para la inclusión de los ítems en los factores hemos mantenido el adoptado por los autores de la escala, se ha incluido el ítem en el factor cuando el peso factorial es superior a .300 .
La prueba Kaiser-Mayer-Olkin de adecuación muestral, $\mathrm{KMO}=.907$, y la prueba de esferidad de Barttlet, $\chi^{2}(406)=$ 9277.41, $p<.000$, indicaron la adecuación de los datos para el análisis. Los cuatro factores explican el $40.76 \%$ de la varianza. En la Tabla 2 se exponen las saturaciones factoriales de cada ítem.

Tabla 1. Media $(M)$, Desviación Típica $(D T)$, Correlación ítem-total corregi$\mathrm{da}\left(r_{d}\right)$ y alfa de Cronbach si el ítem es eliminado $\left(\alpha_{\mathrm{i}}\right)$.

\begin{tabular}{lllll}
\hline Items & $M$ & $D T$ & $r_{d}{ }^{d}$ & $\alpha_{\mathrm{i}}$ \\
\hline GRCS-A1 & 3.57 & 1.66 & .35 & .87 \\
GRCS-A2 & 3.06 & 1.61 & .32 & .87 \\
GRCS-A3 & 2.93 & 1.75 & .38 & .87 \\
GRCS-A4 & 2.95 & 1.75 & .38 & .87 \\
GRCS-A5 & 4.00 & 1.72 & .30 & .87 \\
GRCS-A6 & 2.51 & 1.54 & .33 & .87 \\
GRCS-A7 & 2.76 & 1.72 & .41 & .87 \\
GRCS-A8 & 2.95 & 1.66 & .36 & .87 \\
GRCS-A9 & 3.03 & 1.73 & .44 & .87 \\
GRCS-A10 & 2.83 & 1.81 & .15 & .86 \\
GRCS-A11 & 3.72 & 1.86 & .39 & .87 \\
GRCS-A12 & 2.75 & 1.64 & .29 & .87 \\
GRCS-A13 & 3.94 & 1.68 & .36 & .87 \\
GRCS-A14 & 3.20 & 1.69 & .49 & .86 \\
GRCS-A15 & 3.27 & 1.85 & .39 & .87 \\
GRCS-A16 & 3.89 & 1.70 & .27 & .87 \\
GRCS-A17 & 3.14 & 1.72 & .53 & .86 \\
GRCS-A18 & 3.36 & 1.91 & .45 & .86 \\
GRCS-A19 & 3.52 & 1.79 & .50 & .86 \\
GRCS-A20 & 3.25 & 1.70 & .41 & .86 \\
GRCS-A21 & 3.07 & 1.78 & .46 & .86 \\
GRCS-A22 & 3.18 & 1.89 & .46 & .86 \\
GRCS-A23 & 3.25 & 1.74 & .41 & .86 \\
GRCS-A24 & 3.11 & 1.70 & .44 & .86 \\
GRCS-A25 & 3.40 & 1.82 & .42 & .86 \\
GRCS-A26 & 2.91 & 1.79 & .40 & .87 \\
GRCS-A27 & 3.18 & 1.86 & .39 & .87 \\
GRCS-A28 & 3.14 & 1.74 & .50 & .86 \\
GRCS-A29 & 3.15 & 1.68 & .44 & .86 \\
Total & 93.13 & 23.58 & - & .87 \\
\hline
\end{tabular}

El primer factor estuvo compuesto por 10 ítems que se corresponden con el factor de Emotividad Restringida (RE), que explicó el $22.12 \%$ de la varianza. El segundo factor, Afecto Limitado entre Hombres (RABM), con 7 ítems, explicó el 8.29\% de la varianza. Un $5.43 \%$ de varianza le correspondió al tercer factor, con 6 ítems, Conflicto entre Trabajo, Escuela y Familia (CWSF). El último factor, Necesidad de Éxito y Logro (NSA), con 6 ítems, explicó el $4.76 \%$ de la varianza.

La estructura coincidió con la distribución factorial de la escala original. No obstante, dos ítems modificaron su ubicación factorial original. El item 12 ('Juzgo el valor de otras personas por su nivel de éxito y logro') pasó de la escala de Conflicto entre Trabajo, Escuela y Familia a constituir parte del factor de Necesidad de Éxito y Logro. El ítem 16 ("Es importante para mi hacer las cosas bien en todo momento"), que en la escala original formaba parte de la escala de Necesidad de Éxito y Logro, en nuestro estudio entró a formar parte de la escala de Emotividad Restringida. 
Tabla 2. Solución factorial rotada para los componentes de la GRCS-A en la versión española y comunalidad $\left(b^{2}\right)$ de cada ítem.

\begin{tabular}{|c|c|c|}
\hline \multirow{2}{*}{$\begin{array}{l}\text { Factor e ítem } \\
\text { Emotividad Restringida (RE) }\end{array}$} & \multicolumn{2}{|c|}{ Peso factorial $b^{2}$} \\
\hline & & \\
\hline 17. Encuentro difícil hablar de mis sentimientos con los demás. & .749 & .573 \\
\hline 14. Encuentro difícil expresar a los demás mis necesidades afectivas. & .716 & .514 \\
\hline 19. Me resulta difícil hablar con los demás sobre mis sentimientos intensos. & .711 & .522 \\
\hline 24. No me gusta mostrar mis emociones a otras personas. & .582 & .353 \\
\hline 20. A menudo tengo problemas para encontrar las palabras que describan cómo me siento. & .554 & .337 \\
\hline 9. Expresar mis sentimientos hace que me sienta vulnerable a los ataques de los demás. & .526 & .313 \\
\hline 2. Me resulta difícil decirles a los demás que me preocupo por ellos. & .526 & .302 \\
\hline 29. Cuando me involucro personalmente con los demás, no expreso mis sentimientos más fuertes. & .466 & .307 \\
\hline 16. Es importante para mí hacer las cosas bien en todo momento. & .364 & .205 \\
\hline 6. Me resulta difícil entender las emociones fuertes. & .353 & .157 \\
\hline
\end{tabular}

Afecto Limitado entre Hombres (RABM)

18. Me resulta difícil abrazar a otros hombres.

7. El afecto con otros hombres me pone en tensión.

10. Expresar mis emociones a otros hombres es arriesgado.

21. En algunas ocasiones no me decido a mostrar mi afecto a los hombres por cómo podrían juzgarme los demás. .619 421

26. Intimar mucho con otros hombres me hace sentir incomodo. .618

27. Los hombres que son demasiado simpáticos conmigo hacen que me pregunte sobre sus preferencias sexuales. .614

3. Es difícil para mí expresar de palabra mi cariño a otro hombre.

Conflicto entre Trabajo, Escuela, Familia (CWSF)

22. Mi necesidad de trabajar o estudiar me aparta de mi familia o de mi tiempo libre más de lo que quisiera.

25. Mi trabajo o escuela trastocan a menudo otras partes de mi vida (hogar, salud, tiempo libre).

28. El trabajo excesivo y el estrés a causa de la necesidad de avanzar en el trabajo o en la escuela afecta a

mi vida o la perjudica.

11. Mi carrera, trabajo o escuela influyen en la calidad de mi tiempo libre o vida familiar.

15. Me resulta difícil encontrar tiempo para descansar.

$.717 \quad .530$

4. No me decido entre mi ajetreado trabajo o programa escolar y cuidar de mi salud.

$\begin{array}{ll}.682 & .467 \\ .633 & .403 \\ .504 & .271\end{array}$

Necesidad de Éxito y Logro (NSA)

23. Me esfuerzo por tener más éxito que los demás.

1. Para mí es importante conseguir el primer puesto de mi clase.

8. Algunas veces defino mi valor personal por mi éxito en la escuela.

5. Ganar dinero es parte de mi idea de lo que es ser un hombre de éxito.

13. Me preocupa suspender y cómo afecta esto a que haga las cosas bien como un hombre.

12. Juzgo el valor de otras personas por su nivel de éxito y logro.

\begin{tabular}{ll}
.730 & .539 \\
.678 & .464 \\
.586 & .359 \\
.543 & .348 \\
.534 & .375 \\
.495 & .258 \\
\hline
\end{tabular}

\section{Análisis factorial confirmatorio (AFC)}

Para este análisis se seleccionó al azar el 50\% de la muestra (797 estudiantes). El modelo base para el CRGS-A consistió en la configuración de los cuatro factores extraídos en los análisis factoriales exploratorios. La solución fue satisfactoria con buenos índices de ajuste, GFI $=.93$, AGFI $=.92, \mathrm{CFI}=.96$, RMSEA $=.03$. El modelo se presenta en la Figura 1. La carga de todos los factores fue estadísticamente diferente a cero y en todos los casos superiores a .40, con excepción del ítem 12, que presentó una carga de .36 y del ítem 16 que presentó una carga de .24 .

\section{Consistencia interna}

Los coeficientes de consistencia interna fueron aceptables para todos los factores. Los resultados muestran un alfa de .77 para Emotividad Restringida, .77 para Afectividad Limitada entre Hombres, .78 para Conflicto entre Trabajo, Escuela y Familia y .66 para Necesidad de Éxito y Logro.
Los ítems 12 y 16, que estaban pendientes de revisión, no fueron eliminados del análisis ya que su eliminación no mejoraba la capacidad psicométrica de su subescala. A su vez, a partir del análisis de la consistencia interna, se decidió mantenerlos dentro del nuevo factor en el que saturaron, dado que su ubicación en el factor original de la escala hacía disminuir la fiabilidad de los factores.

\section{Validez concurrente}

Se comprobó la validez concurrente mediante correlaciones de Pearson entre las subescalas de la GRCS-A y las de los otros autoinformes empleados. Tal como se puede apreciar en la Tabla 3, el Conflicto de rol de género presentó correlaciones positivas con todas las escalas (oscilando entre .09 y .32). Los patrones de Conflicto entre el Trabajo, Escuela y Familia y Necesidad de Éxito y Logro se comportaron de manera similar al total de la escala. Por su parte, los patrones de Afecto Limitado entre Hombres y de Emotividad Restringida sólo correlacionaron con la subescalas de sexismo hostil y benévolo. 


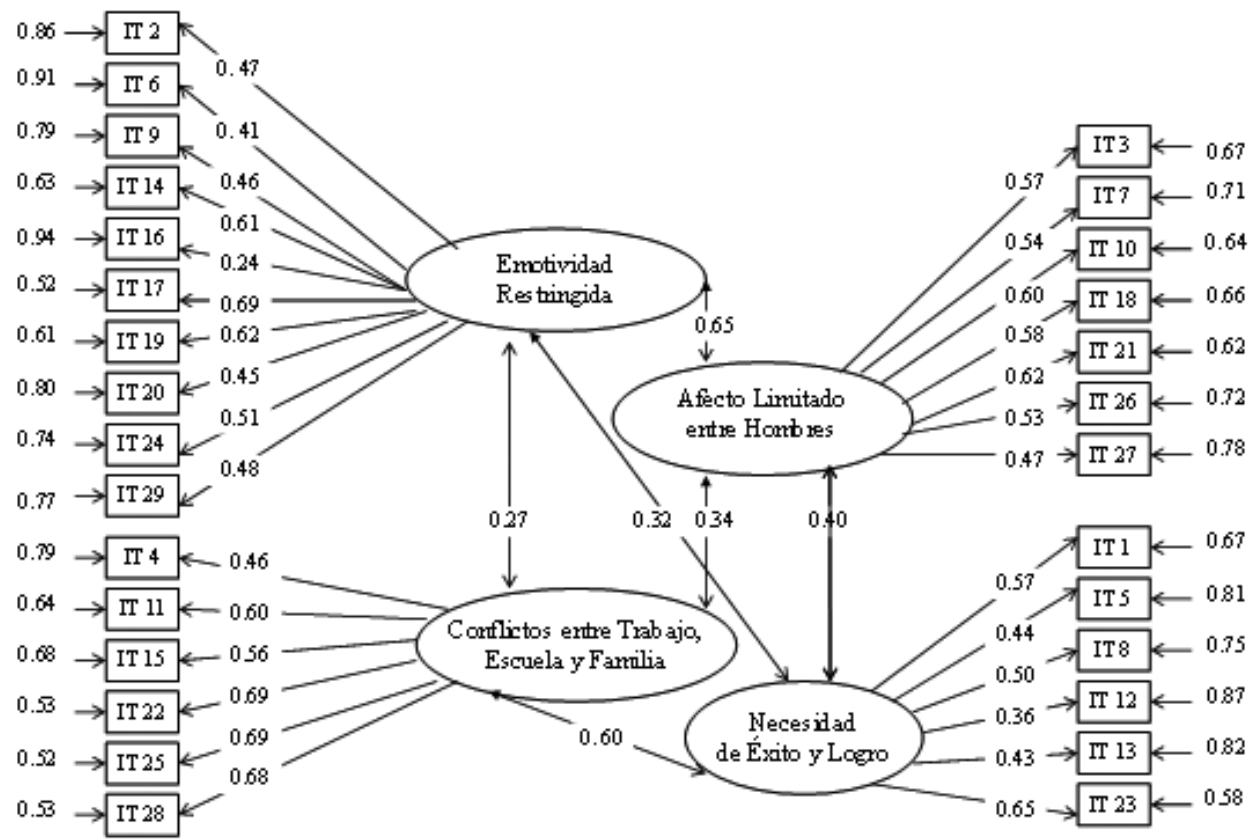

Figura 1. Modelo de análisis factorial confirmatorio de la CRGS-A.

Tabla 3. Correlaciones entre las subescalas del CRGS-A y los factores del ASI y CAME.

\begin{tabular}{|c|c|c|c|c|c|c|}
\hline 2 & 3 & 4 & 5 & 6 & 7 & 8 \\
\hline $.558^{* *}$ & $.391 * *$ & $.402 * *$ & $.836 * *$ & $.197 * *$ & $.209 * *$ & .036 \\
\hline 2 & $.301 * *$ & $.299 * *$ & $.750^{* *}$ & $.293^{* *}$ & $.225^{* *}$ & .040 \\
\hline 3 & & $.424 * *$ & $.695^{* *}$ & $.223^{* *}$ & $.139 * *$ & $.115^{* *}$ \\
\hline 4 & & & $.666^{* *}$ & $.247 * *$ & $.223^{* *}$ & $.087 * *$ \\
\hline 5 & & & & $.318^{* *}$ & $.267 * *$ & $.088^{* *}$ \\
\hline 6 & & & & & $.332^{* *}$ & $.151^{* *}$ \\
\hline
\end{tabular}

Nota. ${ }^{* *} p<.01 .{ }^{*} p<.05$

1= Emotividad Restringida, 2= Afecto Limitado entre Hombres, 3= Conflicto entre Trabajo, Escuela, Familia, 4= Necesidad de Éxito y Logro, $5=$ Conflicto de Rol de Género, $6=$ Sexismo Hostil, $7=$ Sexismo Benévolo, $8=$ Acoso (Rol Agresor).

\section{Influencia de la edad sobre el conflicto de rol de género}

Los datos que se exponen a continuación son los resultados del análisis de regresión lineal realizado tomando como predictora la variable edad. Como puede observarse en la Tabla 4, dos de las cuatro dimensiones entran a formar parte de la predicción del GRC.

Tabla 4. Análisis de regresión sobre el GRC tomando como variable predictora la edad.

\begin{tabular}{llllll}
\hline Paso y variable & $\mathrm{R}^{2}$ & $B$ & $D T B$ & $\beta$ & $t$ \\
\hline $1-\mathrm{RE}$ & .022 & -.229 & .039 & -.150 & $-5.88^{* *}$ \\
& & & & & \\
2- RE & .025 & -.164 & .046 & -.107 & $-3.52^{* *}$ \\
$\mathrm{RABM}$ & & -.099 & .039 & -.078 & $-.257^{* *}$ \\
\hline Nota. ${ }^{* *} p<.01 .{ }^{*} p<.05$ & & & & &
\end{tabular}

Nota. ${ }^{* *} p<.01 . * p<.05$

RE: Emotividad Restringida, RABM: Afecto Limitado entre Hombres

\section{Discusión}

La GRCS-A ha sido diseñada para la evaluación del conflicto de rol de género en adolescentes que, al igual que en los adultos, puede resultar de la desviación o incumplimiento de las normas y roles de género contenidos dentro de la ideología masculina. Se trata de un constructo psicológico de relevancia, si consideramos que la investigación previa ha relacionado el conflicto de rol, entre otras variables, con la ansiedad, la depresión, la agresión sexual, la hostilidad hacia las mujeres y las actitudes negativas hacia la homosexualidad (O’ Neil, 2008). Por este motivo, resulta importante contar con instrumentos válidos y fiables que puedan evaluar cómo influye el aprendizaje del género sobre el bienestar psicológico de los adolescentes y en sus conductas. El objetivo de este estudio fue, primero, traducir la escala original al castellano y examinar sus propiedades psicométricas. Segundo, analizar las correlaciones entre la GRCS-A y las variables psicosociales del sexismo y el acoso escolar y, tercero, examinar las diferencias que se producen de acuerdo a la edad.

En la traducción de la escala se corroboró la equivalencia conceptual del ítem garantizando la comprensión de los adolescentes. Los resultados de nuestra investigación permiten señalar que las propiedades psicométricas de la versión española de la GRCS-A son muy similares a las que presenta la escala original. En lo que se refiere a la estructura factorial, nuestros datos sugieren que la versión castellana de la escala, al igual que su versión inglesa, posee cuatro factores que representan las dimensiones de Emotividad Restringida (RE), 
Afecto Limitado entre Hombres (RABM), Conflicto entre Trabajo, Escuela y Familia (CWSF), Necesidad de Éxito y Logro (NSA). Estos resultados, en términos generales, corroboran los datos sobre las dimensiones identificadas por Blazina et al. (2005). Con respecto a su dimensionalidad, el análisis de componentes principales pone de manifiesto que, a excepción de dos ítems, todos las medidas saturan de forma clara en sus correspondientes dimensiones. En lo que concierne a su consistencia interna, en todos los factores se obtienen altos índices de fiabilidad, constatándose que el Afecto Limitado entre Hombres y el Conflicto entre Trabajo, Escuela y Familia presentan una mayor consistencia interna que en la versión original. Existen, no obstante, algunas diferencias, que comentamos a continuación.

Tal y como se ha indicado, dos ítems de la escala saturan en otro factor, aparte de hacerlo en el factor relevante. Este es el caso de los ítems 12 ("Juzgo el valor de otras personas por su nivel de éxito y logro") y 16 ("Es importante para mí hacer las cosas bien en todo momento"). Por un lado, el ítem 12, aunque pertenece a la dimensión CWSF (Conflicto entre Trabajo, Escuela y Familia), satura de forma significativa en la dimensión NSA (Necesidad de Éxito y Logro). Este mismo efecto ha sido encontrado en la adaptación al coreano de la escala para adolescentes (Kim et al, 2009). Aunque estos resultados deben ser comparados en el futuro con los obtenidos en otras muestras, parecen mostrar que el ítem 12 en la GRCS-A puede ser interpretado como un indicador de la Necesidad de Éxito y Logro en la adolescencia, tal y como sucede en la escala original para adultos (O’Neil et al., 1986). Por otro lado, el ítem 16, perteneciente a la dimensión NSA (Necesidad de Éxito y Logro), satura de forma significativa en el factor RE (Emotividad Restringida). Si se considera su contenido, el ítem 16, no parece que se refiera específicamente a la necesidad de alcanzar determinados logros en la adolescencia, sino que parece reflejar la importancia de ajustarse a las normas sociales en relación con el comportamiento de género.

Otro aspecto a destacar es que la Emotividad Restringida (RE) en nuestro estudio pasa a ser el primer factor de la extracción, explicando más del $20 \%$ de la varianza total. Conviene recordar que la Emotividad Restringida es el factor que más se ha relacionado con problemas psicológicos en adultos (O’Neil, 2002), lo que puede reflejar la relevancia de este factor en los procesos de desarrollo del rol de género.

En cuanto al análisis de la validez concurrente, el patrón de correlaciones encontrado entre las puntuaciones de las dimensiones de la CRGS-A y la medida de sexismo y acoso escolar evidencia la validez de la versión al castellano de este instrumento. La relación entre los distintos patrones de conflicto de rol de género y el sexismo es coherente con la conceptualización teórica. Como afirma O’Neil (2008, p. 367), "las estructuras sexistas de la sociedad y la socialización de rol de género masculino están directamente relacionados con el GRC masculino". Esta relación es congruente con la investigación previa que ha encontrado que el sexismo hostil está relacionado con la experiencia del conflicto de rol de género (Covell, 1998; Schwartz, Tylka y Hood, 2005). Del mismo modo, las correlaciones significativas entre algunos patrones de la versión española de la GRCS-A y el rol de agresor dentro de las dinámicas de acoso escolar son similares a las encontradas en estudios previos con adultos. Concretamente, la investigación ha demostrado que los hombres que experimentan conflicto de rol de género se encuentran más implicados en conductas agresivas y de abuso, como la conducta sexual coercitiva (Seen, Desmarais, Verberg y Wood, 2000), la violencia de género (Breiding, 2004) y la agresión física en contexto experimentales (Cohn, Zeichner y Seibert, 2008). En adolescentes, las investigaciones han destacado que, de forma general, las dificultades en las relaciones interpersonales se asocian con problemas de agresión en el aula (Berger, Rodkin y Dijkstra, 2011; Cerezo y Ato, 2010).

El análisis de regresión revela la influencia de la edad en la Emotividad Restringida y en el Afecto Limitado entre Hombres. Este resultado es congruente con el encontrado en estudios previos, que muestran que durante la adolescencia temprana puede producirse una intensificación en la asimilación de estereotipos, actitudes y conductas de género, como consecuencia del incremento de la presión socializadora para adecuarse a los roles sociales tipificados por sexo (Siverio y García, 2007).

\section{Limitaciones y líneas de investigación futura}

Los resultados obtenidos permiten concluir que la versión en castellano de la GRCS-A ofrece garantías suficientes para una evaluación adecuada del conflicto de rol de género en los varones adolescentes españoles. No obstante, debemos considerar algunas limitaciones que deben ser tenidas en cuenta de cara a la investigación futura sobre las propiedades psicométricas de esta escala.

En primer lugar, la muestra procedía tan solo de dos comunidades autónomas y estuvo compuesta por adolescentes estudiantes en centros de titularidad pública por lo que la generalización de los resultados puede verse limitada. Futuras investigaciones permitirán revisar el instrumento analizando sus propiedades psicométricas en un rango mayor de tipos de centros y en una muestra más heterogénea de adolescentes. En este sentido, la validación de la GRCS-A también debería realizarse con muestras de mujeres tal y como se ha señalado en la investigación sobre su validación a otros idiomas (Kim et al, 2009). Por otra parte, sería interesante estudiar la relación del conflicto de rol de género con las normas de género a través del Inventario de Conformidad con las Normas de Género Masculinas (CMNI) recientemente validado en España (Cuellar-Flores, Sánchez-López y Dresch, 2011). En último lugar, serán necesarias nuevas investigaciones con la GRCS-A relativas a su fiabilidad basada en la estabilidad de las puntuaciones en el tiempo.

Junto a todo ello y considerando la correlación encontrada entre el conflicto de rol de género y, específicamente, los patrones de Conflicto entre Trabajo, Escuela y Familia y 
la Necesidad de Éxito y Logro con el acoso escolar, resulta interesante analizar con mayor profundidad esta la relación con los roles de agresor y víctima, dado que no existen estudios previos que hayan examinado su influencia sobre las conductas agresivas en el contexto escolar. Por último, esperamos que la presente validación de la GRCS-A permita ser utilizada como una herramienta para la evaluación del conflicto de rol de género en adolescentes españoles.

\section{Referencias}

Addelston, J. (1995). Gender role conflicto in elite independent high schools. Unpublished doctoral dissertation. New York: SUNY Graduate School.

Bates-Galligan, S., Barnett, R. V., Brennan, M. A. y Israel, G. (2010). The effects of gender role conflict on adolescent and emerging adult male resiliency. The Journal of Men's Studies, 18, 3-21.

Berger, C., Rodkin, P. C. y Dijkstra, J. K. (2011). Antipathetic relationships among adolescents: Exploring prevalence, gender differences, and stability in the United States and Chile. Anales de Psicologia, 27(3), 783-790.

Blazina, C. (1997). Fear of feminine in the Western psyche and the masculine task of disidentification: Their effects on the development of masculine gender role conflict. The Journal of Men's Studies, 9, 55-68.

Blazina, C. (2001). Gender-role-conflicted men's poor parental attachment and separation/individuation difficulties: Knights without armor in a savage land. The Journal of Men's Studies, 9, 257-265.

Blazina, C., Pisecco, S. y O’Neil, J. M. (2005). An adaptation of gender role conflict scale for adolescents: psychometric issues and correlates with psychological distress. Psychology of Men and Masculinity, 6, 39-45.

Breiding, M. J. (2004). Observed hostility and observed dominance as mediators of the relationship between husbands' gender role conflict and wives' outcomes. Journal of Counseling Psychology, 51, 429-436.

Browne, M. W. y Cudeck, R. (1989). Single sample cross-validation indices for covariance structures. Multivariate Behavioral Research, 24, 445-455.

Browne, M. W. y Cudeck, R. (1993). Alternative ways of assessing model fit. En K. A. Bollen y J. S. Long (Eds.), Testing structural equation models (pp. 136-162). Newbury Park, CA: Sage.

Carretero-Dios, H. y Pérez, C. (2007). Standards for the development and review of instrumental studies: Considerations about test selection in psychological research. International Journal of Clinical and Health Psychology, 7, 863-882.

Cerezo, F. y Ato, M. (2010). Social status, gender, classroom climate and bullying among adolescents pupils. Anales de Psicología, 26(1), 137-144.

Cohn, A. M., Jakupcak, M., Seibert, L. A., Hildebrandt, T. B. y Zeichner, A. (2010). The role of emotion dysregulation in the association between men's restrictive emotionality and use of physical aggression. Psychology of Men and Masculinity, 11, 53-64

Cohn, A. M., Zeichner, A. y Seibert, L. A. (2008). Labile affect as a risk factor for aggressive behaviour in men. Psychology of Men and Masculinity, 9, 29-39.

Covell, A. (1998). Characteristics of college males who are likely to sexually harass women: a test of a mediated model (Doctoral dissertation University of Southern California). Dissertation Abstracts International, 60, 2400.

Cuéllar-Flores, I., Sánchez-López, M. P. y Dresch, V. (2011). El Inventario de Conformidad con las Normas de Género Masculinas (CMNI) en la población española. Anales de Psicología, 27(1), 170-178.

Choi, H., Kim, J., Hwang, M, y Heppner, J. (2010). Self-esteem as a mediator between instrumentality, gender role conflict and depression in male Korean high school students. Sex Roles, 63, 361-372.

Expósito, F., Moya, M. y Glick, P. (1998). Sexismo ambivalente: Medición y correlatos. Revista de Psicología Social, 13, 159-169

Faagot, B. I., Rodgers, C. S. y Leinbach, M. D. (2000). Theories of gender socialization. En T. Eckes y H. M. Trautner (Eds.), The development social psychology of gender (pp. 510-556). Mahwah, NJ: Lawrence Erlbaum.

Glick, P. y Fiske, S. T. (1996). The ambivalent sexism inventory: Differentiating hostile and benevolent sexism. Journal of Personality and Social Psychology, 70, $491-512$.
Agradecimientos.- Esta investigación se ha realizado dentro de Proyecto de Investigación $\mathrm{I}+\mathrm{D}+\mathrm{i}$ "Factores psicosociales de la violencia escolar: la identidad de género como factor diferencial” (Ref. PSI2008-04312/PSI) financiado por el Ministerio de Educación dentro del Plan Nacional de Investigación Científica, Desarrollo e Innovación Tecnológica, 2008-2011.

Groeschel, B. L., Wester, S. R. y Sedivy, S. K. (2010). Gender role conflict, alcohol, and help seeking among college men. Psychology of Men and Masculinity, 11(2), 123-139.

Hambleton, R. K. (1996). Adaptación de test para su uso en diferentes idiomas y culturas: fuentes de error, posibles soluciones y directrices prácticas. En J. Muñiz (Ed.), Psicometría (pp. 203-238). Madrid: Universitas.

Jöreskog, K. G. y Sörbom, D. (1993). LISREL8: Structural Equation Modeling with the simplis command language. Chicago, IL: Scientific Softwarwe.

Kim, J., Choi, H., Kim, H. y Park, K. (2009). Validation of Korean version Gender Role Conflict Scale for Adolescents. Asia Pacific Educational Review, 10, 215-223.

Kiselica, M. S. (2011). Promoting positive masculinity while addressing gender role conflict: A balance theoretical approach to clinical work with boys and men. En C. Blazina y D. Shen-Miller (Eds.), An international psychology of men: Theoretical advances, case studies, and clinical innovations (pp. 127-156). New York: Routledge/Taylor \& Francis Group.

Liang, C. T. H., Salcedo, J. y Miller, H. A. (2011). Perceived racism, masculinity ideologies, and gender role conflicto amnog Latino men. Psychology of Men and Masculinity, 12(3), 201-215.

Marsh, H. W., Balla, J. R. y Hau, K. T. (1996). An evaluation of incremental fit índices: A clarification of mathematical and empirical processes. En G. A. Maroulides y R. E. Schumacker (Eds.), Advanced structural equation modeling techniques (pp. 115-353). Hilsdale, N.J: Erlbaum.

Messick, S. (1995). Standards of validity and the validity standards in performance assessment. Educational Measurement: Issues and Practice, 15, 5 12.

Montero, I. y León, O. G. (2007). A guide for naming research studies in Psychology. International Journal of Clinical and Health Psychology, 7, 847862

Nunnally, J. C. y Bernstein, I. J. (1995). Teoría psicométrica. Madrid: McGrawHill.

O’Neil, J. M., Good, G. E. y Holmes, S. (1995). Fifteen years of theory and research on men's gender role conflict. New paradigms for empirical research. En R.F. Levant y W.S. Pollack (Eds.), A new psychology of men (pp. 164-206). New York: Basic Books.

O’Neil, J. M. (1990). Assessing men's gender role conflict. En D. Moore y F. Leafgren (Eds.), Men in conflict: Problem solving strategies and interventions (pp. 23-38). Alexandria, VA: American Association for Counseling and Development

O’Neil, J. M., Helms, B., Gable, R., David, L. y Wrightsman, L. (1986). Gender Role Conflict Scale (GRCS): College men's fears of femininity. Sex Roles, 14, 335-350.

O’Neil, J. M. (2002). Twenty years of gender role conflict research: Summary of 130 studies. En J. M. O’Neil y G. E. Good (Eds.), Gender role conflict research: Empirical studies and twenty-year summary. $110^{\text {th }}$ Annual Convention of the American Psychological Association, Chicago, IL.

O’Neil, J. M. (2008). Summarizing 25 years of research on men's gender role conflict using the gender role conflict scale: new research paradigms and clinical implications. The Counseling Psychologist, 36, 358-445.

Rigby, K. y Bagshaw, D. (2003). Prospects of adolescent students collaborating with teachers in addressing issues of bullying and conflict in schools. Educational Psychology, 23(5), 535-546.

Robinson, D. T. y Schwartz, J. P. (2004). Relationship between gender role conflict and attitudes toward women and African Americans. Psychology of Men and Masculinity, 5, 65-71. 
Schwartz, J., Tylka, T. L. y Hood, I. B. (2005). Gender role conflict and prejudice: the role of entitlement. En J. M. O’Neil y G.E. Good (Eds), Gender role conflict research studies: Assessing contextual, therapeutic, and interpersonal variables. Symposium conducted at the meeting of the American Psychological Association, Washington, DC.

Senn, C. Y., Desmarais, S., Verberg, N. y Wood, E. (2000). Predicting coercive sexual behaviour across the lifespan in a random sample of Canadian men. Journal of Social and Personal Relationships, 17, 95-113.

Siverio, M. A. y García, M. D. (2007). Autopercepción de adaptación y tristeza en la adolescencia: La influencia del género. Anales de Psicología, 23(1), 41-48.
Steiger, J. H. (1990). Structural model evaluation y modification: An interval stimulation approach. Multivariate Behavioral Research, 25, 173-180.

Tanaka, J. S. y Huba, G. J. (1985). A fit index for covariance structure models under arbitrary GLS estimation. British Journal of Mathematical and Statistical Psychology, 38, 197-201.

Watts, R. H. y Borders, L. D. (2005). Boys' perceptions of the male role: Understanding gender role conflict in adolescent males. Journal of Men's Studies, 13, 267-280.

(Artículo recibido: 16-01-2012, revisado: 12-06-2012, aceptado: 15-06-2012) 